Methods EPIK-O/ENGOT-OV61 (NCT04729387) is a phase 3 , randomized (1:1), open-label, active-controlled trial evaluating the efficacy and safety of alpelisib + olaparib versus single-agent chemotherapy in patients $(\mathrm{N} \approx 358)$ with no germline BRCA mutation, platinum-resistant/refractory HGSOC. Adult women with platinum-resistant/refractory, histologically confirmed HGSOC, high-grade endometrioid ovarian, fallopian tube, or primary peritoneal cancer, with no germline BRCA1/2 mutation, are included; patients must have received 1-3 prior systemic therapies. In Arm 1, patients receive alpelisib $200 \mathrm{mg}$ orally OD + olaparib $200 \mathrm{mg}$ orally BID; in Arm 2, patients receive paclitaxel $80 \mathrm{mg} / \mathrm{m}^{2}$ IV weekly or pegylated liposomal doxorubicin $40-50 \mathrm{mg} / \mathrm{m}^{2}$ IV Q28D (investigator's choice). The primary endpoint is progressionfree survival per radiologic tumor assessment (RECIST 1.1) by a blinded independent review committee. Key secondary endpoint is overall survival. Other secondary endpoints include overall response rate, clinical benefit rate, safety, and quality of life.

Results Enrollment is planned in 28 countries; completion of data collection for the primary endpoint is anticipated in 2023.

Conclusions Not applicable.

\section{EPV280/\#317 A BIZZARE CASE OF ECTOPIC MOLAR PREGNANCY IN BROAD LIGAMENT PROGRESSING TO GTN}

${ }^{1} \mathrm{D}$ Raina*, ${ }^{2} \mathrm{P}$ Tiwari, ${ }^{3} \mathrm{~A}$ Kumari, ${ }^{1,2} \mathrm{D}$ Gupta. ${ }^{1}$ Sumitra Hospital, Gynecology and Obstetrics, Noida, UP, India; ${ }^{2}$ Artemis Hospital, Medical Oncology, Gurugram, Haryana, India; ${ }^{3} \mathrm{Dr}$ Lal Path Labs, Pathology, New Delhi, India

\subsection{6/ijgC-2021-IGCS.351}

Objectives Molar pregnancy occurring at an ectopic site is a rare phenomenon. Such cases are mostly found either in fallopian tubes, uterine cornua or in the ovaries. Only one case of broad ligament molar pregnancy has been reported in literature so far and ours being the first case of a broad ligament ectopic molar pregnancy progressing to GTN. This case report is being presented with the objective of raising the awareness of molar pregnancies occurring at ectopic sites and highlighting the importance of follow-up for such rare cases.

Methods A suspected case of ruptured right tubal ectopic pregnancy presented to emergency with suspiciously high beta HCG level of $85000 \mathrm{mIU} / \mathrm{ml}$. Intraoperatively a distinct mass, separate from uterus and fallopian tube measuring around 8 cms was seen between the leaves of broad ligament. Right salpingo-ophorectomy with excision of broad ligament and right pelvic peritoneum was done. On final histopathology, a diagnosis of broad ligament ectopic complete molar gestation was made.

Results Because of high initial beta HCG levels, large size of ectopic molar mass and fear of losing the patient to followup, prophylactic chemotherapy with single agent methotrexate $50 \mathrm{mg}$ alternating with folinic acid was started. After a brief fall, post surgery, beta HCG started rising for three consecutive weeks despite continued chemotherapy. Gradually with dose modification of methotrexate to $75 \mathrm{mg}$ (6 cycles) she responded and continues to be in remission after seven months.
Conclusions This bizzare case clearly substantiates the existence of such rare conditions and also reinforces the importance of follow-up.

\section{EPV281/\#407 SINGLE-DOSE METHOTREXATE IN THE TREATMENT OF LOW-RISK GESTATIONAL TROPHOBLASTIC NEOPLASIA - AN UPDATED RESULTS}

SF Ngu* KY Tse, MYM Chu, PNH Sheung, KLK Chan. The University of Hong Kong, Queen Mary Hospital, Department of Obstetrics and Gynaecology, Hong Kong, Hong Kong PRC

\subsection{6/ijgc-2021-IGCS.352}

Objectives Low-risk gestational trophoblastic neoplasia (GTN) with WHO prognostic score of 0 to 6 has high cure rate. The aim of the study was to evaluate the effectiveness of single-dose methotrexate infusion in women with low-risk GTN. Methods In this single centre retrospective cohort study, 115 women with low-risk GTN were treated between January 2000 and October 2019 with an intravenous bolus of 100 $\mathrm{mg} / \mathrm{m}^{2}$ of methotrexate followed by a 12-hour infusion of $200 \mathrm{mg} / \mathrm{m}^{2}$. Serum human chorionic gonadotropin (hCG) levels were monitored weekly. If the hCG level dropped by 10 fold after 2 weeks, no further chemotherapy was given. Otherwise, chemotherapy was continued 2-weekly until 3 cycles post-normalisation of hCG. Characteristics between the 2 groups with or without complete remission with this regimen were compared.

Results All 115 women with low-risk GTN were cured. The overall complete remission rate with methotrexate was $85.2 \%$, with $60.9 \%$ of women requiring a single-dose of methotrexate alone, and $24.3 \%$ requiring continuation of chemotherapy with 2-weekly methotrexate. $14.8 \%$ of women had unsatisfactory response with methotrexate alone and were cured with combination of methotrexate and actinomycin-D. The pretreatment hCG levels were significantly lower in women who were cured with single-dose methotrexate regimen compared to those who failed this regimen (median hCG 1227 versus 3335 IU/L; P = 0.037).

Conclusions Single-dose methotrexate regimen offers an effective option for women with low-risk GTN and a low pretreatment hCG level.

\section{EPV282/\#442 CASE REPORT: CHORIOCARCINOMA PRESENTED AS A VAGINAL TUMOR}

${ }^{1} \mathrm{~A}$ Al -Ansari, ${ }^{2} \mathrm{H}$ Khaldi, ${ }^{3} \mathrm{MT}$ Alsayed, ${ }^{4} \mathrm{~S}$ Alhyassat, ${ }^{5} \mathrm{H}$ Almalik, ${ }^{4} \mathrm{~A}$ Al Rashid, ${ }^{4} \mathrm{~S}$ Rashid, ${ }^{2}$ JJO Herod. 'wwrc, Gyn Oncology, Doha, Qatar; ${ }^{2}$ Hamad Medical Corporation, Women Wellness and Research Center, Doha, Qatar; ${ }^{3}$ Hamad Medical Corporation, Women Wellness and Research Center, DOHA, Qatar; ${ }^{4}$ hamad medical coorporation, Pathology, Doha, Qatar; ${ }^{5}$ NCCCR, Oncology, Doha, Qatar

\subsection{6/ijgc-2021-IGCS.353}

Objectives Choriocarcinoma is a highly vascular tumor of the trophoblast with immense metastatic potential to the lung, liver, brain or vulva. Next to the lung, vulvo-vaginal metastasis comprises $30 \%$ of all metastatic incidences. Metastasis in this region is often misleading in its initial appearance. Here we present case of vaginal metastasis of choriocarcinoma which was misdiagnosed initially. 